\title{
Commentary \\ Joint disease caused by defective gp130-mediated STAT signaling
}

\author{
Tetsuji Naka ${ }^{1}$ and Tadamitsu Kishimoto ${ }^{2}$
}

${ }^{1}$ Department of Molecular Medicine, Osaka University Graduate School of Medicine, Osaka, Japan

${ }^{2}$ Osaka University Graduate School, Osaka, Japan

Correspondence: Tetsuji Naka, Osaka University, Graduate School of Medicine, Department of Molecular Medicine, 2-2 Yamadaoka, Suita, Osaka, Japan 565-0871. Tel/fax: +8166879 4143; e-mail: naka@imed3.med.osaka-u.ac.jp

Received: 1 October 2001

Revisions requested: 2 November 2001

Revisions received: 22 November 2001

Accepted: 26 November 2001

Published: 22 January 2002
Arthritis Res 2002, 4:154-156

(C) 2002 BioMed Central Ltd

(Print ISSN 1465-9905; Online ISSN 1465-9913)

\begin{abstract}
IL-6 is a multifunctional cytokine produced by lymphoid and nonlymphoid cells; it regulates immune responses, acute-phase reactions, and inflammation. IL-6 signaling is mediated exclusively by the common signal-transducing component gp130, which is also essential for signal transduction of other cytokines of the leukemia inhibitory factor (LIF)/IL-6 family. M Ernst and colleagues generated and studied knock-in mice (gp130 $\Delta$ STAT/SSTAT), in which all STAT-binding sites (sites binding signal transducers and activators of transcription) were deleted from their gene encoding gp130 but binding sites for both Janus kinases (JAKs) and for the protein tyrosine phosphatase-2 (SHP-2) were preserved. They found that this mutant mouse displayed a blastocyst implantation defect, gastrointestinal ulceration, and, interestingly, severe joint disease with representative features of rheumatoid arthritis. Synovial cells from this mouse exhibited mitogenic hyper-responsiveness to cytokines of the LIF/L-6 family, a phenomenon that was caused by sustained gp130-mediated SHP2/Ras/Erk activation due to a defect in the induction of SOCS-1 (suppressor of cytokine signaling-1; also known as SSI or JAB). This suppressor, induced by STAT signaling, regulates cytokine signaling. It is, therefore, conceivable that the disturbance of the balanced activation between the STAT and

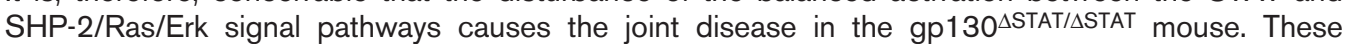
findings may be beneficial in the elucidation of the cause and the treatment of rheumatoid arthritis in humans.
\end{abstract}

Keywords: gp130, interleukin-6, rheumatoid arthritis, suppressor of cytokine signaling

\section{Introduction}

Rheumatoid arthritis (RA) is a major immune-mediated human disease of unknown cause. Nevertheless, cumulative evidence suggests that cytokines are important mediators in its pathology [1]. These substances, especially the so-called proinflammatory cytokines, such as IL-1, tumor necrosis factor- $\alpha$, and IL-6, play a pivotal role in the pathology of RA. Recently, Ohshima et al. showed that IL-6 is essential for the development of antigen-induced arthritis, an experimental autoimmune arthritis model that resembles RA histologically [2]. Furthermore, results of a recent clinical trial of anti-IL-6-receptor antibody therapy for RA support this idea [3] (reviewed in [4]). However, the molecular mechanisms by which IL-6 is involved in joint destruction in RA have not yet been identified. A detailed study of gp130 $\Delta$ STAT/ $\triangle$ STAT mice, in which gp130-mediated signal transducer and activator of transcription (STAT) signal was disrupted, showed that lack of STAT-mediated

IFN = interferon; IL = interleukin; JAK = Janus kinase; LIF = leukemia inhibitory factor; MAPK = mitogen-activated protein kinase; RA = rheumatoid arthritis; SHP-2 = tyrosine phosphatase-2; SOCS = suppressor of cytokine signaling; STAT = signal transducer and activator of transcription. 
induction of suppressor of cytokine signaling-1 (SOCS-1) accelerates the SHP-2/Ras/Erk signal cascade that would be downregulated by SOCS-1 induction; the acceleration eventually resulted in severe joint disease [5].

\section{gp130 signal cascades in IL-6}

The IL-6 receptor consists of two molecules: one is an 80$\mathrm{kDa}$ IL-6-binding protein ( $\alpha$ chain) and the other is a 130$\mathrm{kDa}$ signal transducer, gp130 ( $\beta$ chain), which is shared by the receptors for other cytokines of the IL-6 family, including IL-11, leukemia inhibitory factor (LIF), cardiotrophin-1, oncostatin $\mathrm{M}$, and ciliary neurotrophic factor. IL-6 signaling is exclusively regulated by gp 130 , which contains two motifs conserved among the cytokine receptor family, known as Box1 and Box2 (Fig. 1). The membrane-proximal region containing Box1 and Box2 is sufficient for Janus kinase (JAK) to be activated through gp130. gp130 contains six tyrosines in its cytoplasmic region. Tyr-757 is essential for the tyrosine phosphorylation of SHP-2 and subsequent activation of the Erk mitogen-activated protein kinase (MAPK), while the group of Tyr-765/812/904/914 regulates the binding and activation of STAT1 and STAT3 (see Fig. 1). The STAT3-mediated signal emanating from gp130 is essential and sufficient for the regulation of differentiation and apoptosis of cells and to induce acute-phase proteins [6]. A previous report on a series of knock-in mouse lines, in which gp130-dependent STAT3 and/or SHP-2 signal was disrupted, indicated that gp130-mediated SHP-2 signals not only positively regulate Erk activation but also negatively regulate STAT3 activation [7] (see Fig. 1). It is also known that STAT-mediated signals induce the expression of proteins of the SOCS family, which inhibit JAK-mediated substrate phosphorylation. Thus, the duration and intensity of the signal transmission induced by gp130 are also limited by resulting protein(s) of the SOCS family [8-10] (see Fig. 1). These findings indicate that STAT-mediated signals and SHP-2-mediated signals cross-inhibit each other and presumably provide a balance between these two signalings in vivo. To evaluate the physiological roles of these two gp130-mediated signal cascades, we need to establish mutant mice that can transmit only one signal of these two.

\section{Joint disease in the gp130 $\triangle \mathrm{STAT} / \triangle \mathrm{STAT}$ mouse}

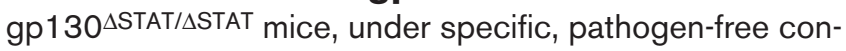
ditions, spontaneously exhibited swelling of one or more of the large, weight-bearing joints and flexion deformities of the bilateral hind legs [5]. These changes occurred young and consistently progressed, albeit with various degrees of severity. The histological examination of clinically affected joints in young gp130 1 STAT/ASTAT mice revealed enlargement of the synovial space, synovial hyperplasia and pannus formation, cartilaginous overgrowth in the menisci, and destruction and deformation of the articular surfaces. In addition, the knee joints of older mutant mice showed destruction and erosion of the articular cartilage
Figure 1

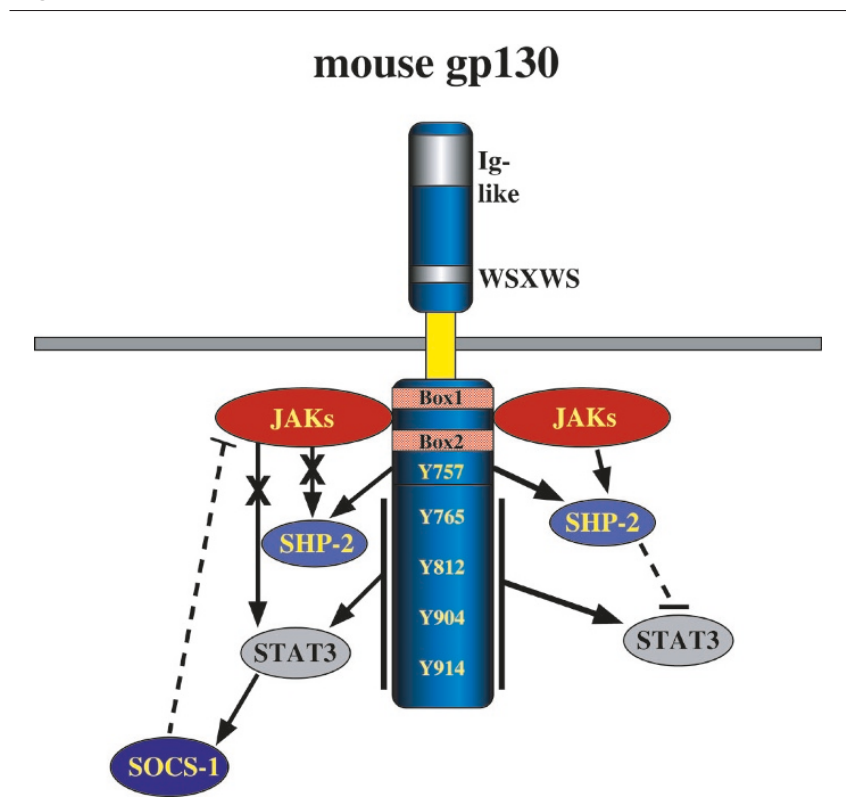

Diagrammatic representation of reciprocal functions of signal cascades through the activation of gp130. The STAT-mediated signal induces the SOCS-1 protein, which is a suppressor of JAKs; and SOCS-1 inhibits both STAT and SHP-2/Ras/Erk mitogen-activated protein kinase activation. On the other hand, the SHP-2-mediated signal inhibits STAT3 activation. JAK = Janus kinase; SHP-2 = tyrosine phosphatase-2; SOCS = suppressor of cytokine signaling; $\mathrm{STAT}=$ signal transducer and activator of transcription.

as well as expansion of the synovial space. Later, inflammatory cells, mostly lymphocytes and macrophages, were found in the synovium and subsynovial tissues of the diseased joints. Taken together, these findings indicate that the joint disease of these mutant mice illustrated destruction of articular cartilage with features of chronic synovitis in various joints, including the major weight-bearing ones.

\section{Molecular mechanism of joint disease in gp130 $\Delta$ STAT/ $\triangle$ STAT mice}

Cartilage destruction associated with synovial hyperplasia is a major event in the early, effector phase of RA. Proinflammatory cytokines, including IL-6, are known to be involved in cartilage destruction in the disease. IL-6 is a potent mitogen for synovial fibroblasts in inflammatory joint diseases, including RA [11]. Mutant synovial fibroblasts derived from the clinically unaffected knee joints exhibited high sensitivity and a proliferative response to IL-6 in comparison with those from control mice. This hyper-responsiveness of the mutant synovial cells to IL- 6 is caused by sustained activation of the SHP-2/Ras/Erk pathway. Transcription of genes of the SOCS family, which are negative regulatory molecules, is activated by STAT-mediated signals. SOCS-1 in particular, which can be induced by 
various cytokines, such as IFN- $\gamma, \mathrm{IL}-4, \mathrm{IL}-2$, and IL-6, inhibits JAK-mediated cytokine signals by binding to JAKs [8-10]. In the mutant synovial cells, SOCS-1 mRNA induction by IL- 6 treatment was markedly less than that in control cells. Moreover, synovial cells of IFN- $\gamma$-deficient SOCS-1-/mice, which are rescued from the perinatal lethality usually associated with SOCS-1 deficiency [12], also displayed sustained SHP-2/Ras/Erk activation by IL-6 stimulation in comparison with control cells. In addition, the synovial cells derived from these doubly mutant mice showed high $\left[{ }^{3} \mathrm{H}\right]$ thymidine incorporation in response to IL-6. Collectively, these findings show that the hyper-responsiveness of gp130 ${ }^{\Delta T A T / \triangle S T A T}$ synovial cells associated with the sustaining of SHP-2/Ras/Erk MAPK activation is due to a lack of SOCS-1 induction, caused by failure of STAT-mediated signals. That is, the disturbed balance between STAT and SHP-2 pathways can be attributed to hyper-responsiveness of synovial cells to IL-6, resulting in joint disease.

\section{Discussion}

The study of gp130 $\Delta$ STAT/ASTAT mice reveals the following two points. First, consistent with previous reports, gp130mediated STAT and SHP-2/Ras/Erk signal pathways play reciprocal roles in immune responses, permitting balanced activation of the two pathways [7,13]. The in vivo findings have elucidated the direct involvement of sustained SHP2/Ras/Erk MAPK activation in joint disease. Second, IL-6 plays a key role in the development of RA [2]. The gp130 $\triangle$ STAT/ASTAT mouse, which we can accept as a model for spontaneously occurring RA, is a useful mouse model for the study of the molecular mechanism of RA and should provide new and important insights for an understanding of the molecular mechanisms in the pathogenesis of RA. However, no one yet knows whether endogenous IFN- $\gamma$ mediated signals are involved in the joint disease of the gp130 $\triangle$ STAT/ASTAT mouse. Hyper-responsiveness of synovial cells from IFN- $\gamma$-deficient SOCS-1/- mice was less marked than in those from gp130 $\triangle \mathrm{STAT} / \triangle \mathrm{STAT}$ mice. Furthermore, previous studies showed that these doubly mutant mice are free from any pathological changes [12,14]. These results suggest that IFN- $\gamma$ may play a role in development of RA in gp130 1 STAT/ASTAT mice. It is worth mentioning that defective induction of SOCS-3 due to lack of STAT3 may contribute to the joint disease of the gp130 $\triangle$ STAT/ASTAT mouse: previous reports showed that SOCS-3 can strongly inhibit IL-6 signaling by competing for the SHP-2-gp130 interaction site by binding to the SH2-domain-containing SHP-2-interaction site in gp130 $[15,16]$. IFN- $\gamma$-deficient gp130 ${ }^{\Delta S T A T / \Delta S T A T}$ mice and conditional SOCS-3-deficient mice will give us these answers.

\section{References}

1. Feldmann M, Brennan FM, Maini RN: Role of cytokines in rheumatoid arthritis. Annu Rev Immunol 1996, 14:397-440.

2. Ohshima S, Saeki Y, Mima T, Sasai M, Nishioka K, Nomura S, Kopf M, Katada Y, Tanaka T, Suemura M, Kishimoto T: Interleukin 6 plays a key role in the development of antigen-induced arthritis. Proc Natl Acad Sci U S A 1998, 95:8222-8226.
3. Yoshizaki K, Nishimoto $N$, Mihara M, Kishimoto T: Therapy of rheumatoid arthritis by blocking IL-6 signal transduction with a humanized anti-IL-6 receptor antibody. Spring semin Immunopathol 1998, 20:247-259.

4. Nishimoto N, Kishimoto T, Yoshizaki K: Anti-interleukin 6 receptor antibody treatment in rheumatic disease. Ann Rheum Dis 2000, 59(suppl):i21-i27.

5. Ernst $M$, Inglese M, Waring $P$, Campbell IK, Bao S, Clay FJ, Alexander WS, Wicks IP, Tarlinton DM, Novak U, Heath JK, Dunn AR: Defective gp130-mediated signal transducer and activator of transcription (STAT) signaling results in degenerative joint disease, gastrointestinal ulceration, and failure of uterine implantation. J Exp Med 2001, 194:189-203.

6. Kishimoto T, Akira S, Narazaki M, Taga T: Interleukin-6 family of cytokines and gp130. Blood 1995, 86:1243-1254.

7. Ohtani T, Ishihara K, Atsumi T, Nishida K, Kaneko Y, Miyata T, Itoh S, Narimatsu M, Maeda H, Fukada T, Itoh M, Okano H, Hibi M, Hirano T: Dissection of signaling cascades through gp130 in vivo: reciprocal roles for STAT3- and SHP2-mediated signals in immune responses. Immunity 2001, 12:95-105.

8. Naka T, Fujimoto $M$, Kishimoto $T$ : Negative regulation of cytokine signaling: STAT-induced STAT inhibitor. Trends Biochem Sci 1999, 24:394-398.

9. Yasukawa $H$, Sasaki A, Yoshimura A: Negative regulation of cytokine signaling pathways. Annu Rev Immuno/ 2000, 18:143164.

10. Krebs DL, Hilton DJ: SOCS proteins: negative regulators of cytokine signaling. Stem Cells 2001, 19:378-387.

11. Mihara M, Moriya $Y$, Kishimoto $T$, Ohsugi $Y$ : Interleukin-6 (IL-6) induces the proliferation of synovial fibroblastic cells in the presence of soluble IL-6 receptor. Br J Rheumatol 1995, 34: 321-325

12. Marine JC, Topham DJ, MacKay C, Wang D, Parganas E, Stravopodis D, Yoshimura A, Ihle JN: SOCS1 deficiency causes a lymphocyte-dependent perinatal lethality. Cell 1999, 98:609616.

13. Symes A, Stahl N, Reeves SA, Farruggella T, Servidei T, Gearan T, Yancopoulos G, Fink JS: The protein tyrosine phosphatase SHP-2 negatively regulates ciliary neurotrophic factor induction of gene expression. Curr Biol 1997, 7:697-700.

14. Alexander WS, Starr R, Fenner JE, Scott CL, Handman E, Sprigg NS, Corbin JE, Cornish AL, Darwiche R, Owczarek CM, Kay TW, Nicola NA, Hertzog PJ, Metcalf D, Hilton DJ: SOCS1 is a critical inhibitor of interferon gamma signaling and prevents the potentially fatal neonatal actions of this cytokine. Cell 1999, 98:597-608.

15. Nicholson SE, De Souza D, Fabri LJ, Corbin J, Willson TA, Zhang JG, Silva A, Asimakis M, Farley A, Nash AD, Metcalf D, Hilton DJ, Nicola NA, Baca M: Suppressor of cytokine signaling-3 preferentially binds to the SHP-2-binding site on the shared cytokine receptor subunit gp130. Proc Natl Acad Sci U S A 2000, 97:6493-6498.

16. Schmitz J, Weissenbach M, Haan S, Heinrich PC, Schaper F: SOCS3 exerts its inhibitory function on interleukin- 6 signal transduction through the SHP2 recruitment site of gp130. J Biol Chem 2000, 275:12848-12856. 